\title{
OBSERVATORIO DEL HÁBITAT DE BOGOTÁ: Hacia una propuesta para su creación en la Universidad La Gran Colombia
}

\author{
Juan Carlos Marín Villegas \\ Universidad La Gran Colombia, UGC \\ juan.marin@ugc.edu.co
}

\section{RESUMEN}

El presente escrito se deriva de un estudio que busca plantear una propuesta para la creación del "Observatorio del Hábitat de Bogotá", OHB, en la Facultad de Arquitectura de la Universidad La Gran Colombia, UGC. Después de una reflexión conceptual y teórica sobre los observatorios, el hábitat y su sostenibilidad y de una contextualización acerca de la situación del hábitat y su discusión en el ámbito político internacional, el artículo presenta los principales resultados del proceso de investigación que fundamenta la propuesta: la descripción y análisis del estado de la observación del hábitat en el contexto mundial, nacional y local, de metodologías y experiencias en la planeación e implementación de observatorios de similar orientación , y de los lineamientos institucionales de la UGC relacionados con la temática en estudio; por último y con base en la interpretación de estos resultados, se presentan aspectos básicos del diseño del OHB.

Palabras clave: Observatorio académico, Hábitat humano, Desarrollo sostenible

\begin{abstract}
This letter is derived from a study that seeks to raise a proposal for the creation of the "Habitat Observatory of Bogota", OHB, at the Faculty of Architecture of the La Gran Colombia University, UGC. After a conceptual and theoretical reflection on observatories, habitat and its sustainability and a contextualization about the habitat situation and its discussion in the international political arena, the article presents the main results of the research process that underlies the proposal: the description and analysis of the state of observation habitat at the global, national and local context, methodologies and experience in planning and implementing Observatories of similar orientation, and institutional guidelines of the UGC related to the topic under study. Finally, based on the interpretation of these results, the basic aspects of the design of the OHB are presented.
\end{abstract}

Key words: Academic Observatory, Human Habitat, Sustainability development 


\section{INTRODUCCIÓN}

La crítica situación de los asentamientos humanos, principalmente en países en vía de desarrollo y los graves problemas ambientales por los que atraviesa el planeta, han convertido a la sostenibilidad en uno de los principales paradigmas contemporáneos en la gestión del hábitat humano. En el desarrollo sostenible la necesidad de información se presenta en todos los niveles, desde el de las personas encargadas de la toma de decisiones a nivel superior, hasta el nivel popular e individual (Strong, 1998). En Latino América la gestión de la información urbana y territorial es un tema emergente. La profesionalización y el manejo de los discursos técnicos sobre los indicadores, los temas de la agenda pública, los procesos de gestión y el financiamiento se adecuan en la mayoría de los casos a un programa establecido por la ONU a través del programa Hábitat y que se ha institucionalizado en varios países con el fin de monitorear las ciudades en torno a distintas agendas internacionales (Frausto, Soijet y Chacón, 2013).

En vista de la inexistencia en Bogotá de un observatorio que haga parte de esta iniciativa mundial, y en razón a que por tanto la ciudad región adolece de una organización cuyo trabajo le permita compararse en el contexto internacional, así como evaluar su situación y avances respecto a lo planteado en Agendas como la de Hábitat y los Objetivos de Desarrollo Sostenible, la Facultad de Arquitectura de la Universidad La Gran Colombia, buscando además mecanismos que le sirvan para fomentar la formación investigativa, el trabajo en red, la colaboración dinámica institucional e interinstitucional y la proyección hacia las comunidades más vulnerables, se ha propuesto la creación y puesta en marcha del "Observatorio del Hábitat de Bogotá". Así entonces, desde la misma facultad se viene adelantando un estudio cuyo objetivo es plantear el diseño de esta organización, a partir de la reflexión teórica y conceptual, de un diagnóstico de las necesidades del momento, los procesos explicativos o generadores involucrados y de las tendencias futuras.

\subsection{Los observatorios, el hábitat humano y su sostenibilidad}

Los observatorios son organismos creados por un colectivo, con el fin de seguir la evolución de un fenómeno desde una posición ventajosa (Enjunto, 2008, citado en Marcial, 2009). Para ello, estos instrumentos cumplen con tres funciones básicas: en primer lugar actúan como un centro de documentación, encargándose de monitorear, recopilar, tratar y difundir información; además, fungen como un foro permanente en el que distintos actores estratégicos adelantan el análisis, evaluación y discusión no sólo de indicadores, sino también de políticas, legislación y buenas prácticas; por último, operan como órganos consultivos, en la medida en que formulan sugerencias y posibles correcciones (Husillos, 2006, citado en Marcial, 2009; Pardo y Méndez, 2014). Las funciones básicas que desarrolla un observatorio implican en su organización la existencia como mínimo de cuatro áreas: un área técnica, en la cual se encuentra el Sistema de Información -SI- y el Sistema de Información Geográfica -Sig-; una investigativa, donde se hace la lectura y la interpretación de la información procesada por el Sig y donde se lidera la formulación de sugerencias y correcciones; una de difusión, encargada de presentar ante la opinión pública los resultados del trabajo de conjunto entre el Sig y el área de investigación, y adicionalmente debe contar con un área administrativa (Vicepresidencia de la República de Colombia, 2006; Correa y Castellanos, 2014).

En cuanto al hábitat humano, este puede entenderse como un sistema multidimensional propio del habitar, de la estancia del hombre en la tierra. El hábitat humano comprende el ámbito en el cual los sujetos (individuales y colectivos) establecen redes de relaciones con unas condiciones materiales de naturaleza biótica, física y antrópica; las cuales, a partir de sus intercambios, hacen posible diversas formas de habitar y de producción de técnicas que de manera permanente configuran y transforman sus hábitos y las formas de organización de su habitar (Sánchez, 2003). Para Marín (2014), el hábitat humano puede entenderse como un sistema ambiental vivo y por lo tanto complejo de interrelaciones ecosistema - cultura, en el que se desarrollan procesos de construcción material y simbólica, en escalas que van desde la vivienda hasta la región.

En relación al desarrollo sostenible, la aproximación de la Organización de Naciones Unidas, ONU, que lo entiende como aquel que "garantiza las necesidades del presente sin comprometer las posibilidades de las generaciones futuras para satisfacer sus propias necesidades" (World Commission on Environment and Development, 1992), constituye uno de los referentes centrales de la discusión sobre el tema. Desde los 90's, distintos estudios se han dedicado a precisar y ampliar las dimensiones y escalas asociadas al desarrollo sostenible, conduciendo a entenderlo en términos de la interacción equilibrada de dinámicas ambientales, 
humanas e incluso político-institucionales (Galindo, 2012). Es así que el desarrollo sostenible del hábitat humano, puede definirse como un proceso de integración sinérgica y de coevolución entre las dimensiones de las que este se compone, que garantiza un nivel no decreciente de bienestar a la población a largo plazo, sin comprometer las posibilidades de desarrollo de las áreas vecinas y contribuyendo a la reducción de los efectos nocivos de las transformaciones adaptativas de la cultura sobre los ecosistemas. Este planteamiento implica trascender preocupaciones relacionadas exclusivamente con el crecimiento económico y urbano cuantitativo, aspectos a los que se redujo el desarrollo en su sentido clásico, hacia objetivos que en todas las dimensiones propendan por hábitats más habitables, en los que exista una relación más armoniosa con el medio ambiente natural y en los que, por ende, el desarrollo humano sea la principal preocupación.

\subsection{El hábitat y su discusión en el contexto político internacional}

En 1950, una tercera parte de la población mundial vivía en ciudades. Ante las dificultades para satisfacer las necesidades y aspiraciones básicas de las poblaciones, en 1976 fue organizada por la ONU la primera de las conferencias internacionales sobre asentamientos humanos, Hábitat I, en Vancouver, Canadá. En esta cumbre se advirtió que la condición de los asentamientos humanos determina en gran medida la calidad de la vida, cuyo mejoramiento es indispensable para la satisfacción plena de las necesidades básicas, por lo que se adoptaron políticas para la creación de asentamientos humanos más habitables, considerándolos como instrumentos y objetos del desarrollo.

Para finales del siglo XX, la proporción de población urbana había aumentado ya a la mitad. El crecimiento acelerado de las áreas urbanas limitó la capacidad de las ciudades para atender las necesidades de la población urbana, lo que se manifestó en el incremento del desempleo, el empleo informal, acceso limitado a la vivienda por parte de los sectores más desprotegidos, un mayor impacto al medio ambiente, y problemas de acceso a los servicios de salud y educación. En ese contexto, en 1996 se llevó a cabo Hábitat II, en Estambul, Turquía. Los dos temas principales del encuentro fueron "la vivienda adecuada para todos" y "el desarrollo de asentamientos humanos sostenibles en un mundo en proceso de urbanización". Como resultado de esta Conferencia, se adoptó la Agenda Hábitat, documento que sería utilizado como una guía para la acción y un instrumento para el monitoreo de sus avances. En el encuentro se declaró que una vivienda adecuada significa disponer de un lugar privado, espacio suficiente, accesibilidad física, seguridad adecuada, seguridad de tenencia, estabilidad y durabilidad estructurales, iluminación, calefacción y ventilación suficientes, una infraestructura básica adecuada que incluya servicios de abastecimiento de agua, saneamiento y eliminación de desechos, factores apropiados de calidad del medio ambiente y relacionados (Habitat I.I., 1996). Respecto al objetivo "Asentamientos humanos sostenibles en un mundo en proceso de urbanización", los Jefes de Estado y de Gobierno se comprometieron a desarrollar sociedades que hagan uso eficiente de los recursos dentro de los límites de la capacidad de carga de los ecosistemas y ofrezcan a todas las personas, en particular las que pertenecen a grupos vulnerables y desfavorecidos, las mismas oportunidades de llevar una vida sana, segura y productiva en armonía con la naturaleza y su patrimonio cultural y valores espirituales y culturales, y que garanticen el desarrollo económico y social y la protección del medio ambiente (Habitat l.I., 1996).

Desde 2009 la mayoría de la población mundial vive en ciudades. Actualmente, las ciudades son espacios donde la pobreza multidimensional, la degradación ambiental, la vulnerabilidad a los desastres y el impacto del cambio climático están presentes. Ante esta situación, en los Objetivos de Desarrollo Sostenible, adoptados en 2015 por los miembros de la ONU como agenda posterior a los Objetivos de Desarrollo del Milenio, se estableció el Objetivo 11: "Lograr que las ciudades y los asentamientos humanos sean inclusivos, seguros, resilientes y sostenibles", el cual contempla metas relacionadas con la vivienda adecuada, transporte adecuado, planificación y gestión participativa, protección del patrimonio, reducción de afectación por desastres, reducción del impacto ambiental, acceso universal a zonas verdes y espacios públicos, vínculos positivos entre zonas urbanas y rurales, políticas y planes integrados y edificios sostenibles y resilientes.

Más recientemente, en octubre de 2016, en la Tercera Conferencia sobre Vivienda y Desarrollo urbano Sostenible, Hábitat III, los estados miembro de la ONU adoptaron la Nueva Agenda Urbana, NAU, la cual se estructura a través de compromisos en torno a las tres dimensiones del desarrollo sostenible: el desarrollo urbano sostenible para la inclusión social y la erradicación de la pobreza, la prosperidad urbana sostenible e inclusiva y oportunidades para todos y el desarrollo urbano resiliente y sostenible ambientalmente. El Plan de ejecución de Quito para la NAU prevé tres ejes para la realización de un desarrollo urbano sostenible: 1. Los 
compromisos transformadores para el desarrollo urbano sostenible: a través de la equidad urbana y erradicación de la pobreza sin olvidar a nadie y de la prosperidad urbana sostenible, inclusiva y de oportunidades para todos. 2. Implementación efectiva: a través de la construcción de la estructura urbana; de la planificación y administración del desarrollo urbano espacial y del mejoramiento de los medios de implementación de la NAU. 3. Seguimiento y revisión de las acciones e implementación de la NAU en los países miembros de Naciones Unidas (ONU-Habitat, 2016).

\section{METODOLOGÍA}

La investigación se adelanta desde un enfoque cualitativo y de tipo proyectivo: con base en los resultados de un proceso investigativo se precisan lineamientos y orientaciones para la creación y puesta en marcha del "Observatorio del Hábitat de Bogotá". Los tres primeros objetivos específicos del estudio son entonces: la descripción y análisis (1) del estado de la observación del hábitat en el contexto internacional, nacional y local, (2) de metodologías y experiencias en el diseño, implementación y funcionamiento de observatorios de similar orientación y (3) de las políticas, planes, programas y proyectos institucionales relacionados con el tema. Para el desarrollo de estos objetivos se utiliza como método la heurística, esto es la búsqueda y compilación de diversas fuentes de información relacionadas con el tema, y posteriormente la hermenéutica, mediante la lectura, análisis, interpretación, correlación y clasificación de la información, según el grado de interés y necesidad frente a la investigación. Las técnicas utilizadas son entonces bibliográficas y el instrumento manejado consiste en fichas para la sistematización de la información.

\section{RESULTADOS}

\subsection{La observación del hábitat en el contexto mundial, nacional y local}

Para dar seguimiento al progreso en la implementación de la Agenda Hábitat, desde 1997 fue establecido por ONU-Habitat el Observatorio Urbano Global, GUO. Como esquema ideal para su funcionamiento, se propuso la creación de una Red de Observatorios Urbanos Nacionales, que aportarían elementos de análisis y reflexión, alimentados a su vez en cada país por una red de Observatorios Urbanos Locales (OULs). Coordinados por el GUO, los objetivos de la Red de Observatorios Urbanos son, según ONU-Hábitat (s.f.): estimular procesos de consulta para ayudar a identificar e integrar las necesidades urbanas de información; ayudar a desarrollar capacidades para la recolección, manejo y aplicaciones de política de información urbana, centrada en indicadores y mejores prácticas; proveer información y análisis a todos los interesados para lograr una participación más efectiva en la toma de decisiones urbanas; compartir información y conocimientos especializados usando tecnología e infraestructura de información modernas.

Para el seguimiento de la Agenda Hábitat se establecieron 42 indicadores: 20 Indicadores clave (IC), 9 Checklist (CL) y 13 Indicadores Extensivos (IE). Los indicadores se clasifican en dos grupos: A, formado por indicadores que se obtienen de los censos generales de población y de las encuestas nacionales de hogares; $B$, que se pueden obtener de otras fuentes: registros oficiales, estudios publicados por instituciones de gobierno, empresas paraestatales, organizaciones no gubernamentales, académicas, instituciones financieras y también el uso de estimaciones de grupos de expertos. 
Capítulo de la Agenda Hábitat

Promover el Derecho a Vivienda Adecuada

Posibilitar la seguridad de la Tenencia

Promover igualdad de acceso a créditos Proporcionar igualdad de acceso a la tierra

Promover el acceso a los servicios básicos

Promover la igualdad de oportunidades para una vida sana y segura

Promover la integración social y apoyar a los grupos desfavorecidos

Promover la igualdad de género en el desarrollo de asentamientos humanos

Promover una estructura geográficamente equilibrada de los asentamientos humanos

Administrar el suministro y demanda de agua de forma eficaz

Reducir la contaminación en zonas urbanas

Prevenir desastres y reconstruir los asentamientos

Promover sistemas de transporte eficaces y ambientalmente racionales

Fortalecer las microempresas y pequeñas empresas, particularmente las establecidas por mujeres

Fomentar las asociaciones de los sectores

público y privado y estimular las

oportunidades de empleo productivo

Promover la descentralización y fortalecer las autoridades locales

Fomentar y apoyar la participación y el compromiso cívico

Garantizar la administración transparente, responsable y eficaz de pueblos, ciudades y zonas metropolitanas

Indicadores de la Agenda Hábitat

(ONU-Habitat / Rolac, 2004).
Indicadores

Grupo

IC1: Estructuras durables

IC2: Área suficiente para vivir

CL1: Derecho a Vivienda Adecuada

IE1: Precio de Vivienda y coeficiente arrendo-ingreso

IC3: Tenencia Segura

IE2: Vivienda autorizada

IE3: Desalojos

CL2: Financiamiento de Vivienda

IE4: Relación precio de tierra e ingreso

IC4: Acceso a agua potable

IC5: Acceso a saneamiento mejorada

IC6: Conexiones domiciliarias

IC7: Mortalidad Infantil

IC8: Homicidio

CL3: Violencia Urbana

IE5: HIV incidencia

IC9: Hogares pobres

IC10: Tasa de alfabetización

CL4: Inclusión de Genero

IE6: Tasa de escolaridad

IE7: Concejalas féminas

IC11: Crecimiento Población Urbana

IC12: Asentamientos planificados

IC13: Precio de Agua

IE8: Consumo de agua

IC14: Aguas residuales tratadas:

IC15: Eliminación de Desechos sólidos

IE9: Recolección regular de desechos sólidos

CL5: Prevención de Desastres e instrumentos de

mitigación

IE10: Viviendas en ubicación sujeta a riesgos

IC16: Tiempo de traslado

IE11: Medios de transporte

Planes ambientales locales

IC17: Empleo Informal

IC18: Producto Urbano

IC19: Desempleo

IC20: Ingresos de Gobiernos Locales

CL7: Descentralización

CL8: Participación ciudadana

IE12: Participación electores

IE13: Asociaciones Civiles

CL9: Transparencia y responsabilidad
A-B

B

A-B

B

A

A

A

A

B

B

A-B

A

A

B

A

B

A

B

B

B

B

B

B

B

B

B

B

$\mathrm{B}$

-B

B

$B$

B

B

B

B

B 
Actualmente, el GUO lleva a cabo además actividades relacionadas al seguimiento del ODS 11, a través de un conjunto de indicadores globales acordados en la Comisión de Estadísticas de la ONU en 2016. El programa actualiza regularmente los indicadores urbanos, produce una base de datos de indicadores urbanos globales, y coordina el uso de indicadores urbanos.

\begin{tabular}{|c|c|c|}
\hline Tema & Indicador & Número \\
\hline $\begin{array}{l}\text { Viviendas, servicios } \\
\text { básicos y barrios } \\
\text { marginales. }\end{array}$ & $\begin{array}{l}\text { Porcentaje de la población urbana que vive en barrios marginales } \\
\text { o asentamientos informales (Indicador ODM) }\end{array}$ & 66 \\
\hline $\begin{array}{l}\text { Sistemas de } \\
\text { transporte }\end{array}$ & Porcentaje de personas a menos de $0,5 \mathrm{~km}$ del transporte público & 67 \\
\hline $\begin{array}{l}\text { Urbanización } \\
\text { inclusiva y } \\
\text { sostenible }\end{array}$ & $\begin{array}{l}\text { Relación entre la tasa de consumo de suelo a la tasa de } \\
\text { crecimiento de la población, en escala comparable que se } \\
\text { desarrolló. }\end{array}$ & 68 \\
\hline Desastres & $\begin{array}{l}\text { Pérdidas por desastres naturales, por el clima y los eventos no } \\
\text { relacionados con el clima (en US } \$ \text { y en vidas perdidas) }\end{array}$ & $\begin{array}{l}6 \text { de referencias } \\
\text { cruzadas }\end{array}$ \\
\hline Impacto ambiental & $\begin{array}{l}\text { Media de la contaminación del aire urbano por material } \\
\text { particulado (PM10 y PM2.5) }\end{array}$ & 69 \\
\hline $\begin{array}{l}\text { Zonas verdes y } \\
\text { espacios públicos }\end{array}$ & $\begin{array}{l}\text { Área del espacio público y verde como una proporción de la } \\
\text { superficie total de la ciudad }\end{array}$ & 70 \\
\hline Impacto ambiental & $\begin{array}{l}\text { Porcentaje de residuos sólidos urbanos recogidos regularmente y } \\
\text { bien administrados }\end{array}$ & 71 \\
\hline $\begin{array}{l}\text { Políticas y planes } \\
\text { integrados }\end{array}$ & $\begin{array}{l}\text { Ingresos nacionales asignados al desarrollo sostenible como } \\
\text { porcentaje del PIB, por sectores }\end{array}$ & $\begin{array}{l}95 \text { de referencias } \\
\text { cruzadas }\end{array}$ \\
\hline $\begin{array}{l}\text { Zonas verdes y } \\
\text { espacios públicos }\end{array}$ & Número de intersecciones de calles por kilómetro cuadrado & $\begin{array}{c}\text { Nacional } \\
\text { complementario }\end{array}$ \\
\hline $\begin{array}{l}\text { Políticas y planes } \\
\text { integrados }\end{array}$ & $\begin{array}{l}\text { Existencia y aplicación de un marco nacional de política de } \\
\text { asentamientos urbanos y humanos }\end{array}$ & $\begin{array}{c}\text { Nacional } \\
\text { complementario }\end{array}$ \\
\hline $\begin{array}{l}\text { Políticas y planes } \\
\text { integrados }\end{array}$ & $\begin{array}{l}\text { Porcentaje de ciudades con más de } 100.000 \text { habitantes, que } \\
\text { están implementando las estrategias de reducción de riesgos y } \\
\text { resiliencia informados por marcos internacionales aceptados }\end{array}$ & $\begin{array}{l}\text { Nacional } \\
\text { complementario }\end{array}$ \\
\hline $\begin{array}{l}\text { Edificios } \\
\text { sostenibles y } \\
\text { resilientes }\end{array}$ & $\begin{array}{l}\text { Presencia de los códigos de construcción urbanos, ya sea que } \\
\text { estipulan el uso de materiales locales y/o nuevas tecnologías de } \\
\text { eficiencia de energía o con incentivos para el mismo }\end{array}$ & $\begin{array}{l}\text { Nacional } \\
\text { complementario }\end{array}$ \\
\hline $\begin{array}{l}\text { Patrimonio cultural } \\
\text { y natural }\end{array}$ & Índice de biodiversidad de la ciudad (índice de Singapur) & $\begin{array}{c}\text { Nacional } \\
\text { complementario }\end{array}$ \\
\hline $\begin{array}{l}\text { Vínculos entre las } \\
\text { zonas rurales y } \\
\text { urbanas }\end{array}$ & $\begin{array}{l}\text { Porcentaje de consumo de alimentos y materias primas dentro de } \\
\text { las áreas urbanas que son producidos y entregados en/desde las } \\
\text { zonas rurales dentro del país }\end{array}$ & $\begin{array}{c}\text { Nacional } \\
\text { complementario }\end{array}$ \\
\hline
\end{tabular}

Indicadores mundiales de seguimiento del Objetivo 11 de los ODS.

Elaboración propia con base en información de S.D.S.N. (2015).

En 2012, ONU-Habitat creó el Índice de Prosperidad de las Ciudades, IPC, como una herramienta para medir la sostenibilidad de las ciudades y el nivel de prosperidad que generan en los habitantes. EI IPC se concentra en ciudades de forma individual y mide la prosperidad a partir de las dimensiones de productividad, infraestructura, calidad de vida, equidad y sostenibilidad ambiental, con la gobernanza urbana como elemento transversal articulador. EI IPC se calcula de manera incremental en tres modalidades: el índice básico (IPCb) que incluye las variables mínimas que permiten hacer comparaciones entre las ciudades y entre países (26 indicadores); el índice extendido (IPCe) que agrega otras variables que se consideran relevantes, pero cuya información no está disponible en todas las ciudades del mundo (56 indicadores); el índice contextual (IPCc) que incorpora toda aquella información que permite comprender las realidades particulares de cada territorio (ONU-Hábitat Colombia, 2015). 


\begin{tabular}{|c|c|c|}
\hline Dimensión & Subdimensión & Indicador \\
\hline \multirow{7}{*}{ Productividad } & \multirow{3}{*}{ 1.Crecimiento Económico } & 1. PIB Per Cápita (Básico) \\
\hline & & 2. Dependencia Adultos Mayores (Básico) \\
\hline & & 3. Ingreso Medio de los Hogares \\
\hline & $\begin{array}{ll}2 . & \text { Aglomeración } \\
\text { Económica } & \end{array}$ & 1. Densidad Económica (Básico) \\
\hline & \multirow{3}{*}{ Empleo } & 1. Tasa de Desempleo (Básico) \\
\hline & & 2. Relación Empleo-Población \\
\hline & & 3. Empleo Informal \\
\hline \multirow{19}{*}{ Infraestructura } & \multirow{6}{*}{$\begin{array}{l}\text { 1. Infraestructura de } \\
\text { Vivienda }\end{array}$} & 1. Vivienda Durable (básico) \\
\hline & & 2. Acceso a Agua Mejorada (básico) \\
\hline & & 3. Acceso a Saneamiento Adecuado \\
\hline & & 4. Acceso a Electricidad \\
\hline & & 5. Espacio Vital Suficiente (básico) \\
\hline & & 6. Densidad Residencial \\
\hline & \multirow{2}{*}{ 2. Infraestructura Social } & 1. Densidad de Médicos (básico) \\
\hline & & 2. Número de Bibliotecas Públicas \\
\hline & \multirow{3}{*}{ 3. ICT } & 1. Acceso a Internet (básico) \\
\hline & & 2. Acceso a Computadoras \\
\hline & & 3. Velocidad Promedio de Banda Ancha \\
\hline & \multirow{5}{*}{ 4. Movilidad Urbana } & 1. Uso del Transporte Público (básico) \\
\hline & & 2. Tiempo Promedio de Viaje Diario (básico) \\
\hline & & 3. Longitud del sistema de transporte masivo \\
\hline & & 4. Fatalidad por Accidentes de Tránsito \\
\hline & & 5. Asequibilidad del Transporte \\
\hline & \multirow{3}{*}{ 5. Conectividad de las Vías } & 1. Densidad de la Interconexión Vial (básico) \\
\hline & & 2. Densidad Vial (básico) \\
\hline & & 3. Superficie Destinada a Vías (básico) \\
\hline \multirow{13}{*}{ Calidad de Vida } & \multirow{4}{*}{ 1. Salud } & 1. Esperanza de Vida al Nacer (básico) \\
\hline & & 2. Tasa de Mortalidad de Menores de Cinco Años (básico) \\
\hline & & 3. Cobertura Vacunación \\
\hline & & 4. Mortalidad Materna \\
\hline & \multirow{5}{*}{ 2. Educación } & 1. Tasa de Alfabetización (básico) \\
\hline & & 2. Promedio de Años de Escolaridad (básico) \\
\hline & & $\begin{array}{l}\text { 3. Participación de menores de } 6 \text { años en Programas de } \\
\text { Desarrollo de la Primera Infancia }\end{array}$ \\
\hline & & 4. Tasa Neta de Matrícula en Educación Superior \\
\hline & & 5. Número de Universidades Top \\
\hline & \multirow{2}{*}{ 3. Seguridad y Protección } & 1. Tasa de Homicidios (básico) \\
\hline & & 2. Tasa de Hurtos \\
\hline & \multirow{2}{*}{ 4. Espacio Público } & 1. Accesibilidad al Espacio Público Abierto \\
\hline & & 2. Área Verde per cápita (básico) \\
\hline \multirow{7}{*}{$\begin{array}{l}\text { Equidad e } \\
\text { Inclusión Social }\end{array}$} & \multirow{2}{*}{ 1. Equidad Económica } & 1. Coeficiente de Gini (básico) \\
\hline & & 2. Tasa de Pobreza (básico) \\
\hline & \multirow{2}{*}{ 2. Inclusión Social } & 1. Viviendas en Tugurios (básico) \\
\hline & & 2. Desempleo Juvenil (básico) \\
\hline & \multirow{3}{*}{ 3. Inclusión de Género } & 1. Inscripción Equitativa en Educación de Nivel Secundario \\
\hline & & 2. Mujeres en los Gobiernos Locales \\
\hline & & 3. Mujeres en el Mercado Laboral \\
\hline \multirow{5}{*}{$\begin{array}{l}\text { Sostenibilidad } \\
\text { Ambiental }\end{array}$} & \multirow{3}{*}{ 1. Calidad del Aire } & 1. Número de Estaciones de Monitoreo (básico) \\
\hline & & 2. Concentración de PM10 \\
\hline & & 3. Emisiones de CO2 \\
\hline & \multirow{2}{*}{ 2. Manejo de Residuos } & 1. Recolección de Residuos Sólidos (básico) \\
\hline & & 2. Tratamiento de Aguas Residuales (básico) \\
\hline
\end{tabular}




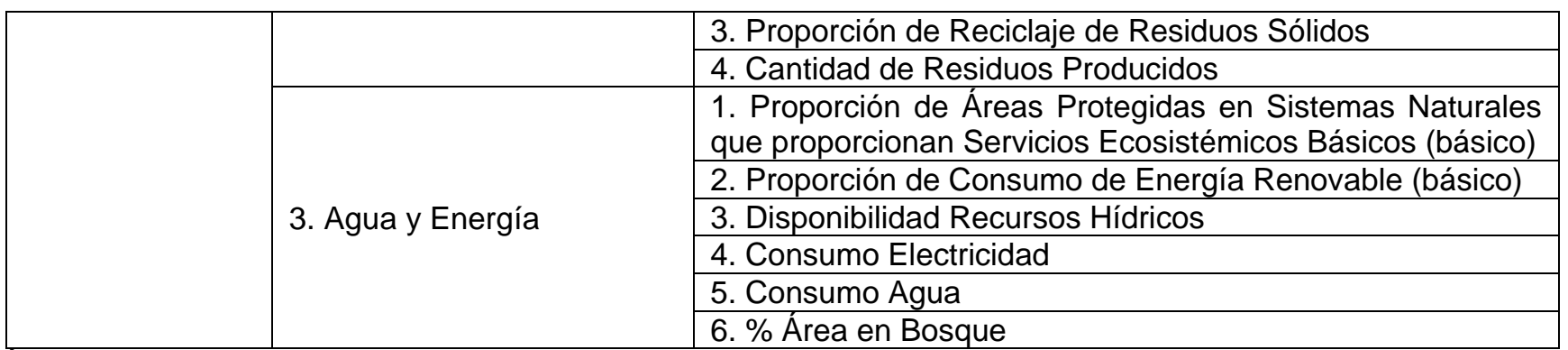

Índice de Prosperidad de las Ciudades

(ONU-Habitat Colombia, 2015)

ONU-Habitat, en alianza con distintas entidades nacionales, tomó la determinación de aplicar en Colombia el IPC y realizar el "Primer Reporte sobre el Estado de las Ciudades de Colombia", presentado a finales de 2015 como uno de los primeros ejercicios de esta naturaleza a nivel mundial. El reporte utiliza como fuente principal la Encuesta Continua de Hogares del DANE y hace uso de otras encuestas oficiales, registros administrativos e información suministrada directamente por las ciudades. A partir de esta experiencia, Findeter, el Fondo Nacional del Ahorro y la oficina de ONU-Hábitat en el país, dieron inicio a mediados de 2016 a un proceso que pretende articular a diversos actores con el fin de dar vida al "Observatorio Urbano de Colombia", OUC, como órgano consultivo y centro de referencia en temáticas relacionadas con políticas públicas de desarrollo territorial. Según ONU-Habitat Colombia (2016), se espera que aunando esfuerzos con los diferentes sectores que intervienen en el desarrollo urbano y territorial, se logre consolidar un instrumento que vaya en línea con las disposiciones de la NAU y los ODS. La metodología sobre datos del OUC giraría en torno, por una parte, al enriquecimiento del IPC, y por otro lado, al seguimiento de los indicadores de los ODS.

Además de lo anterior, en el país existen otras dos iniciativas para la Observación urbana y territorial que actualmente vienen haciendo trámite en cabeza del Departamento Nacional de Planeación: El Observatorio del Sistema de ciudades, OSC, el cual se encuentra ya formulado (con una metodología de indicadores propios) y en proceso de implementación, y el Observatorio Nacional de Ordenamiento Territorial, el cual se articularía al primero. La creación del OSC obedece al mandato del CONPES 3819 de 2014, el cual establece un plan de acción que contempla, entre otros, la implementación por parte de DNP de un Observatorio a partir de las aglomeraciones urbanas, con el fin de generar información para la toma de decisiones de política pública y del Plan Nacional de Desarrollo 2014- 2018, que contempla la implementación del Observatorio, con el fin de realizar seguimiento a la Política del Sistema de Ciudades y apoyar en el ordenamiento del territorio para la sostenibilidad, el desarrollo y la equidad territorial en municipios, departamentos y áreas metropolitanas, a partir de la compilación y articulación de información para la toma de decisiones. Por su parte, la iniciativa de creación del Observatorio Nacional de Ordenamiento Territorial, responde al mandato del Decreto 3680 de 2011, en el que se señala la necesidad de promover la creación de un observatorio que cuente con información que permita soportar técnicamente las evaluaciones, las revisiones y las sugerencias que se formulen al Gobierno Nacional y a las Comisiones Especiales de Seguimiento al Proceso de Descentralización y Ordenamiento Territorial del Senado de la República y de la Cámara de Representantes para la adopción de políticas, desarrollos legislativos y criterios para la mejor organización del Estado en el territorio (Art. 7).

En el contexto local de Bogotá, desde hace varios años, la administración distrital ha motivado la creación de un número considerable de observatorios enfocados en diferentes temas, en cabeza de distintos sectores del gobierno de la ciudad y encargados de hacer seguimiento fundamentalmente a las correspondientes políticas públicas sectoriales. Varios de estos presentan un trabajo dinámico y otros se encuentran inactivos o quedaron formulados y sin implementación. La naturaleza de estos es netamente pública, en la mayoría de los casos, aunque algunos trabajan de manera asociada con la academia (Pardo y Méndez, 2014). 


\begin{tabular}{|c|c|c|}
\hline $\begin{array}{l}\text { Observatorio } \\
\text { Ambiental de Bogotá } \\
\text { (Secretaría del } \\
\text { Ambiente) }\end{array}$ & $\begin{array}{l}\text { Conocer a través de indicadores ambientales el } \\
\text { estado y la calidad del ambiente en Bogotá, así } \\
\text { como los resultados de la gestión desarrollada por } \\
\text { varias entidades del Sistema Ambiental del Distrito } \\
\text { Capital (SIAC) frente a problemas ambientales del } \\
\text { Distrito Capital. }\end{array}$ & $\begin{array}{l}\text { Cambio climático, control para } \\
\text { la calidad ambiental, } \\
\text { ecosistemas, educación } \\
\text { ambiental y participación, } \\
\text { gestión ambiental empresarial, } \\
\text { movilidad sostenible, } \\
\text { ordenamiento } \\
\begin{array}{l}\text { Ecourbanismo, ruralidad. y } \\
\end{array}\end{array}$ \\
\hline $\begin{array}{l}\text { Observatorio rural de } \\
\text { Bogotá (Secretaría } \\
\text { del Ambiente) }\end{array}$ & $\begin{array}{l}\text { Integrar información básica sobre el estado } \\
\text { ambiental, social, cultural, económico e institucional } \\
\text { de la ruralidad del Distrito Capital, con el fin de } \\
\text { apoyar la gestión y planeación del desarrollo rural }\end{array}$ & $\begin{array}{l}\text { Ejes ambiental, social, cultural, } \\
\text { económico e institucional de la } \\
\text { ruralidad del Distrito Capital. }\end{array}$ \\
\hline $\begin{array}{l}\text { Observatorio de } \\
\text { Salud Ambiental de } \\
\text { Bogotá (Secretaría } \\
\text { de Salud) }\end{array}$ & $\begin{array}{l}\text { Integrar, promover y apoyar esfuerzos distritales de } \\
\text { carácter técnico, político y social tendientes al } \\
\text { mejoramiento y conservación del ambiente como } \\
\text { estrategia para mejorar las condiciones de salud y } \\
\text { la calidad de vida de los bogotanos. }\end{array}$ & $\begin{array}{l}\text { Aire, ruido y radiación; } \\
\text { medicamentos seguros; } \\
\text { calidad de agua y } \\
\text { saneamiento básico; cambio } \\
\text { climático; alimentos sanos y } \\
\text { seguros; hábitat, espacio } \\
\text { público y movilidad; eventos } \\
\text { transmisibles }\end{array}$ \\
\hline $\begin{array}{l}\text { Observatorio para la } \\
\text { Equidad en Calidad } \\
\text { de Vida y Salud de } \\
\text { Bogotá, D.C } \\
\text { (Secretaría de Salud) }\end{array}$ & $\begin{array}{l}\text { Medir, analizar, divulgar y propiciar el debate } \\
\text { público, promover la formulación de políticas } \\
\text { públicas para la superación de las inequidades en } \\
\text { salud, el mejoramiento de la calidad de vida y de } \\
\text { las condiciones ambientales, y divulgar la } \\
\text { información que se genere para que sea útil a los } \\
\text { tomadores de decisiones, servidores públicos, } \\
\text { comunidad y técnicos. }\end{array}$ & $\begin{array}{l}\text { Calidad de vida urbana, riesgo } \\
\text { y protección, desigualdad en el } \\
\text { acceso a servicios de salud y } \\
\text { protección. }\end{array}$ \\
\hline $\begin{array}{l}\text { Observatorio } \\
\text { Dinámicas del } \\
\text { Territorio (Secretaría } \\
\text { de Planeación) }\end{array}$ & $\begin{array}{l}\text { Recopila, organiza, administra, produce y analiza la } \\
\text { información relacionada con aspectos económicos, } \\
\text { sociales, demográficos, ambientales, territoriales y } \\
\text { de competitividad, requerida para la formulación, } \\
\text { adopción, ejecución, seguimiento y evaluación de } \\
\text { los instrumentos de planeación del territorio. }\end{array}$ & $\begin{array}{l}\text { Mercado de la vivienda } \\
\text { principalmente }\end{array}$ \\
\hline $\begin{array}{l}\text { Observatorio de la } \\
\text { dinámica urbano } \\
\text { territorial (Secretaría } \\
\text { de Planeación) }\end{array}$ & $\begin{array}{l}\text { Realizar el seguimiento y la evaluación de la } \\
\text { dinámica urbano-regional (Bogotá y } 31 \text { municipios } \\
\text { de Cundinamarca) en diferentes escalas } \\
\text { territoriales para permitir la estructuración de } \\
\text { políticas públicas (formulado y sin implementar). }\end{array}$ & $\begin{array}{l}4 \text { ejes temáticos (económico, } \\
\text { social, institucional y ambiente } \\
\text { territorial) }\end{array}$ \\
\hline $\begin{array}{l}\text { Observatorio Distrital } \\
\text { de Gestión de } \\
\text { Riesgos y Cambio } \\
\text { Climático- ODGR-CC } \\
\text { (IDIGER) }\end{array}$ & $\begin{array}{l}\text { Generación de conocimiento sobre los riesgos y su } \\
\text { gestión a través de la recopilación, el análisis y la } \\
\text { interpretación de la información correspondiente y } \\
\text { el seguimiento estadístico de las principales } \\
\text { variables que dan cuenta de los riesgos existentes } \\
\text { (condición de riesgos) y de los esfuerzos distritales } \\
\text { por reducirlo y controlarlo (gestión). }\end{array}$ & $\begin{array}{l}\text { Condiciones de riesgo y } \\
\text { efectos del Cambio Climático; } \\
\text { percepción de riesgos; gestión } \\
\text { de riesgos; mitigación y } \\
\text { adaptación al cambio climático }\end{array}$ \\
\hline $\begin{array}{l}\text { Observatorio Técnico } \\
\text { Catastral (Catastro } \\
\text { Distrital) }\end{array}$ & $\begin{array}{l}\text { Generar conocimiento relacionado con la actividad } \\
\text { catastral mediante el estudio de la información } \\
\text { predial a partir del análisis de resultados de } \\
\text { procesos catastrales, líneas de investigación e } \\
\text { identificación de mejores prácticas cuyos } \\
\text { resultados permitan ser una herramienta de apoyo } \\
\text { para la toma de decisiones en los diferentes } \\
\text { ámbitos multipropósitos. }\end{array}$ & $\begin{array}{l}\text { Desarrollo } \\
\text { desarrollo físico y dinámica } \\
\text { urbana; desarrollo jurídico; } \\
\text { desarrollo cartográfico y } \\
\text { espacial; gestión normativa }\end{array}$ \\
\hline
\end{tabular}




\begin{tabular}{|c|c|c|}
\hline $\begin{array}{l}\text { Observatorio de } \\
\text { Desarrollo } \\
\text { Económico } \\
\text { (Secretaría de } \\
\text { Desarrollo } \\
\text { económico) }\end{array}$ & $\begin{array}{l}\text { Generar estadística y análisis sobre sectores y } \\
\text { variables asociados con el desarrollo económico de } \\
\text { Bogotá. }\end{array}$ & $\begin{array}{l}\text { Comercio al por } \\
\text { menor, Comercio } \\
\text { exterior, Competitividad, Const } \\
\text { rucción, Consumo y } \\
\text { precios, Dinámica económica y } \\
\text { distribución, Emprendimiento y } \\
\text { Microfinanzas, Industria, Segur } \\
\text { idad alimentaria y } \\
\text { ruralidad, Servicios, Trabajo } \\
\text { decente y digno }\end{array}$ \\
\hline $\begin{array}{c}\text { Observatorio de } \\
\text { Culturas (Secretaría } \\
\text { de cultura recreación } \\
\text { y deporte) }\end{array}$ & $\begin{array}{l}\text { Generar conocimiento de ciudad, útil para la } \\
\text { formulación, seguimiento y diseño de políticas } \\
\text { públicas }\end{array}$ & $\begin{array}{llr}\text { Actividades públicas que } \\
\text { promueve y organiza la } \\
\text { Secretaría de Cultura, } \\
\text { Recreación y Deporte y sus } \\
\text { entidades adscritas }\end{array}$ \\
\hline
\end{tabular}

Principales observatorios públicos sectoriales de Bogotá D.C.

Elaboración propia

Por otra parte, la Cámara de Comercio de Bogotá, ofrece a la ciudadanía en general información sobre el desarrollo urbano, el espacio público y la movilidad de Bogotá y la región, por medio de publicaciones, boletines y estudios. Sin embargo, esta entidad no cuenta con un equipo específico que "observa", sino que diversifica el análisis de información en distintos grupos de la organización, los cuales toman como fuente primaria los datos suministrados por las entidades públicas (Sanabria y Méndez, 2014). En la ciudad existen además dos iniciativas surgidas desde ámbitos académicos: los observatorios locales, creados por el Instituto de Estudios Urbanos de la Universidad Nacional de Colombia con el apoyo de las localidades de Engativá, Teusaquillo, Chapinero y Tunjuelito, como un instrumento que divulga información relevante para llevar a cabo el monitoreo y el seguimiento de las condiciones económicas, sociales, urbanas y de gestión del gobierno a escala local y vecinal y el "Observatorio Urbano Hábitat", unidad adscrita a la Facultad de Ciencias del Hábitat de Universidad de La Salle, cuya misión es promover la interacción e integración de los saberes académicos y las necesidades concretas de la sociedad entorno a los problemas del Hábitat (Sanabria y Méndez, 2014).

\subsection{Formulación, diseño e implementación de Observatorios: metodologías y experiencias}

\subsubsection{La metodología de ONU-Hábitat}

El Observatorio Urbano Global alienta la designación de OULs como instituciones de carácter urbano para involucrar en el diálogo a los hacedores de políticas locales y organizaciones de la sociedad civil, para generar información sobre temas y problemas locales y para alentar respuestas de política a las necesidades y prioridades experimentadas localmente. Según ONU-Habitat (s.f.), establecer un OUL no implica crear un nuevo organismo, pues en la mayoría de los casos una entidad existente puede asumir sus funciones. Para ONUHabitat Costa Rica (2010), en la implementación de un OUL se deben seguir seis fases: 1. Interés en formar parte de un observatorio como resultado de un consenso participativo; 2 . Elaboración de la propuesta de temas y objetivos del observatorio; 3. Formalización de acuerdos, estructura, reglamentos, recursos, funciones y aspectos necesarios para la implementación del observatorio; 4. Recopilación, diagnóstico y sistematización de la información; 5. Implementación y consolidación del observatorio; 6 . Análisis y divulgación de la información. Para el diseño de los observatorios vinculados a la Red de OULs, el GUO especifica tres componentes básicos: i. un sistema de indicadores estratégicos sobre la ciudad; ii. un sistema de información, que mantenga disponibles los indicadores; y, iii. un componente de gestión, que promueva a los observatorios como espacios de análisis y reflexión. La red del OUG ofrece a los OULs herramientas y beneficios, como acceso a préstamos para adquisición de equipos y herramientas, capacitación, acceso a información, conferencias de miembros de la red, entre otros.

\subsubsection{La experiencia del Observatorio Urbano de Córdoba y del Metrosum de Toluca}

El Observatorio Urbano Córdoba (Argentina), OUC, fue puesto en marcha en el año 2004 a partir de la iniciativa de un conjunto de unidades académicas de la Universidad Nacional de Córdoba, con un foco de análisis 
centrado en la ciudad y su entorno metropolitano. El propósito del OUC es "desarrollar una base de datos a escala urbana y metropolitana con información cuantitativa y cualitativa actualizada, confiable, oportuna y accesible, en términos territoriales, ambientales, sociales, políticos y económicos, que permita contar con un conocimiento amplio de la ciudad metropolitana y de su evolución en el tiempo" (OUC, s.f.). La estructura organizativa del OUC se compone de cinco áreas: operaciones, encargada del relevamiento, procesamiento, carga de datos e informes; investigaciones, donde se realizan diagnósticos, evaluaciones e investigaciones; difusión, encargada de prensa y comunicación; técnica dedicada a la asistencia informática; y administrativa, cuya función principal es la del manejo de recursos económicos.

Por su parte, el Observatorio Metropolitano de Toluca (México), Metrosum, fue creado en 2005 por investigadores del Colegio Mexiquense y de la Universidad Autónoma del Estado de México, con la misión de "Contribuir con el desarrollo de la Zona Metropolitana de Toluca (ZMVT), sumando esfuerzos con distintos actores sociales, públicos y privados, organizando y monitoreando un sistema de indicadores, produciendo estudios, análisis y conocimiento, apoyando procesos de reflexión y de toma de decisiones sobre la ciudad" (Metrosum, s.f.). La primera etapa de trabajo del Observatorio consistió en definir un detallado protocolo metodológico que guiara la recolección y el ordenamiento de la información, lo que permitió contar con información que cumple, al menos, con cinco requisitos básicos de calidad definidos por el equipo investigador: 1) relevante, 2) confiable, 3) comparable, 4) rastreable y 5) auditable (Chávez, Álvarez y Garrocho, 2007). Para facilitar comparaciones intraurbanas, Metrosum contiene información de la ZMVT desde la escala metropolitana, pasando por la municipal y de localidad, hasta la de colonia o AGEB (Área Geoestadística Básica). El observatorio dispone de un sistema de información distribuido con componentes geográficos que facilita manipular la información de territorios concretos (a diferentes escalas de agregación), agilizar su consulta y despliegue (tabular, gráfico y cartográfico) y simplificar su análisis. Una condición importante en el diseño de Metrosum fue encontrar soluciones tecnológicas de bajo costo y aplicación general. Para garantizar el libre acceso a su información se cuenta con una página web con una interfaz amigable que simplifica su operación.

Ambos observatorios fueron creados siguiendo la metodología general propuesta por ONU-Hábitat (con las adecuaciones hechas para México por la Secretaría de Desarrollo Social en el caso de Metrosum). En los dos casos también, la metodología base se adecuó a las condiciones de la ciudad y su área metropolitana y a la disponibilidad de información. Tanto en el OUC como el Metrosum se establecieron indicadores adicionales atendiendo a las necesidades y problemáticas específicas, a través de foros de discusión con actores locales en el caso del primero y mediante un trabajo de investigadores expertos en el segundo.

Para Chávez, Álvarez y Garrocho (2007), "la principal debilidad de Metrosum se localiza en su vertiente de gestión. El equipo técnico y académico que diseñó y construyó el observatorio no ha tenido la capacidad de vincular al observatorio con el sector público ni con los sectores privado y social". Por esto, los mismos autores señalan que es recomendable que desde el inicio del diseño de los observatorios urbanos se incorpore a los diversos sectores de la sociedad interesados en el desarrollo urbano local, con el fin de fortalecer la plataforma de apoyo social para los observatorios e incrementar su efecto y utilidad real. Este ha sido el caso del OUC, pues según la misma organización, las actividades desarrolladas por parte del equipo de trabajo, han permitido la articulación de una red de actores interesados en promover acciones para el desarrollo sustentable y calidad de vida, sumándolos en la provisión de información, la recopilación de datos y construcción de Indicadores. Desde 2004, el Observatorio trabaja en convenio con la Municipalidad de Córdoba y desde 2006 con la Gerencia de Estadísticas de la ciudad. Sin embargo, según el OUC, en su operación se ha tenido que hacer frente a la falta de adecuación de la estructura de las organizaciones públicas para la recolección, procesamiento y difusión de información con estándares de calidad adecuados, a una baja o nula propensión de los funcionarios públicos para tomar decisiones informadas y a la ausencia de una cultura política que valore la importancia del monitoreo y la evaluación de las políticas públicas, así como de la rendición de cuentas de las acciones públicas a los ciudadanos (OUC, s.f.).

\subsection{Lineamientos institucionales de la UGC}

A partir de la docencia, la investigación y la proyección social, La Universidad la Gran Colombia busca ser un poder de cuestionamiento y crítica de las estructuras, valores y comportamientos vigentes, formulando respuestas a los problemas sociales y elaborando modelos y proyectos de nueva sociedad o civilización, mediante un compromiso de investigación-acción-participación (UGC, 2016a). En correspondencia con los 
propósitos de formación y en el marco de la Misión y Visión institucional, el Programa de Arquitectura propugna por formar profesionales con sensibilidad para desarrollar sus proyectos con responsabilidad social en procura del mejoramiento de la calidad de vida de las comunidades. Por esta razón, el diseño curricular del programa parte de la selección de problemas de la realidad con vistas a su transformación, de manera que en sus tres núcleos de formación se incluyen ejes temáticos relacionados principalmente con el proyecto integral local, zonal y metropolitano, con la intervención patrimonial y con la vivienda social (Facultad de Arquitectura, 2016). También, a partir del antecedente del "Observatorio de Mejoramiento Urbano", el cual se propuso la recolección y procesamiento de información para la identificación de las características, dinámicas, valores y comportamientos de los asentamientos informales y su interacción e impacto sobre el resto de la ciudad, en la línea de formación investigativa del programa se trabaja alrededor de problemas asociados a sectores de protección ambiental, con declaratoria patrimonial y en estado de marginalidad.

En cuanto a investigación, actualmente la UGC establece la línea institucional "Humanismo hacia lo sostenible y valores sociales solidarios". En consecuencia, la facultad de Arquitectura ha definido como línea central de investigación el Hábitat, como problema principal de investigación la formación y desarrollo del Hábitat Humano y como líneas primarias de investigación las de Hábitat Sociocultural, Diseño y Gestión del Hábitat Territorial y Hábitat Tecnológico y Construcción, dentro de las cuales se articulan los grupos y los semilleros de investigación. Según la Facultad de Arquitectura (2010), la implementación de las Líneas Primarias debe gestionarse en los escenarios de La Universidad, el Estado y la sociedad y la Empresa. En cuanto a la universidad, se plantea la participación en redes de información y conocimiento generadas por otras organizaciones, universidades y facultades nacionales e internacionales, con el ánimo de enriquecer la perspectiva propia y proveer puntos de vista a otros actores del proceso de construcción del Hábitat. En relación con el Estado y la sociedad, el conocimiento generado desde los Programa de la Facultad de Arquitectura debe gestionarse hacia entidades gubernamentales y grupos sociales con el ánimo de ofrecer acompañamiento profesional y especializado que favorezca la toma de decisiones y la participación de los sectores sociales en la construcción del Hábitat.

En relación con la proyección social, según el Proyecto Educativo Institucional, las necesidades sociales deben ser convertidas en problemas académicos para que la academia pueda enfrentarlas con sus propias herramientas, con el fin de crear un vínculo indisoluble con la investigación (UGC, 2016a). En el eje estratégico 2 "Una universidad socialmente responsable" del Plan Estratégico institucional de Desarrollo 2016-2020, la UGC se planteó como objetivo central, "aportar de manera efectiva en el proceso de transformación de la sociedad a través de soluciones emanadas de una conciencia social, articuladas mediante la solidaridad, la cooperación y la reciprocidad, la búsqueda del bien común garantizando la alineación de personas y procesos" (UGC, 2016b). De esta manera, la UGG y su Facultad de Arquitectura se han planteado la reflexión y praxis para el compromiso social como una de sus principales políticas, la cual debe llevarse a cabo mediante estrategias como el desarrollo de proyectos para la atención de las necesidades de la comunidad y la búsqueda de mecanismos de cooperación externa para el desarrollo de proyectos conjuntos e intercambio de experiencias (Facultad de Arquitectura, 2011).

\subsection{Observatorio del Hábitat de Bogotá, OHB}

De acuerdo con lo señalado, se plantea que el Observatorio del Hábitat de Bogotá, OHB, asuma como objetivo general: monitorear y evaluar la sostenibilidad del hábitat urbano regional de Bogotá, mediante la gestión integrada de sistemas de información, análisis y socialización, para la promoción de la reflexión, el intercambio de conocimiento y la formulación de políticas, planes, programas y proyectos de intervención. Así entonces, como objetivos específicos del OHB se proponen: 1. Monitorear la situación de las distintas dimensiones que en conjunto conforman el hábitat urbano regional de Bogotá, a través de la recopilación y tratamiento de información cualitativa y cuantitativa relevante; 2. Promover la reflexión y el intercambio de conocimiento mediante el análisis, evaluación y difusión de indicadores, políticas, legislación y buenas prácticas por parte de distintos actores estratégicos de la ciudad región: comunidad académica, representantes de la sociedad civil, tomadores de decisiones en el sector público, entre otros; 3. Formular políticas, planes, programas y proyectos de intervención que den respuesta a problemáticas urbano-regionales a través de la realización de estudios e investigaciones aplicadas. 
Derivado de lo anterior, se proyecta que el OHB tenga como funciones: Trabajar con grupos asociados para desarrollar y aplicar indicadores, índices y mecanismos de evaluación adecuados; Mantener sistemas de manejo de información y realizar evaluaciones y análisis de impacto a petición de las autoridades y los grupos de socios; Desarrollar capacidades para la generación, manejo, análisis y difusión de información, incluyendo información empírica, de manera regular y consistente; Identificar condiciones, tendencias y temas prioritarios por medio de la investigación y los procesos consultivos en los que participan funcionarios y organizaciones de la sociedad civil; Proponer opciones para armonizar las políticas y estrategias sectoriales; Cooperar con otros Observatorios para compartir recursos, intercambiar conocimientos substantivos y metodológicos y difundir información en los ámbitos nacional, regional y global; Analizar y compartir lecciones aprendidas de experiencias en marcha y buenas prácticas; Mantener una página de Internet y un boletín de noticias para brindar información sobre el hábitat y para reportar sobre las actividades del Observatorio y sus grupos de socios; Producir un informe bienal sobre el Estado de la Ciudad, que incluirá análisis comparativos de indicadores y una presentación de mejores prácticas.

Para el cumplimiento de los objetivos propuestos, se postula que la observación desde el OHB se concentre en los temas y subtemas correspondientes a las dimensiones ambiental natural, tecnológica y físico-espacial, socio cultural e histórica y política y económica del hábitat urbano regional de Bogotá, que coinciden con los aspectos clave de la NAU, el ODS 11, el IPC y con las prioridades del ámbito de observación y los intereses propios de la UGC y su Facultad de Arquitectura.

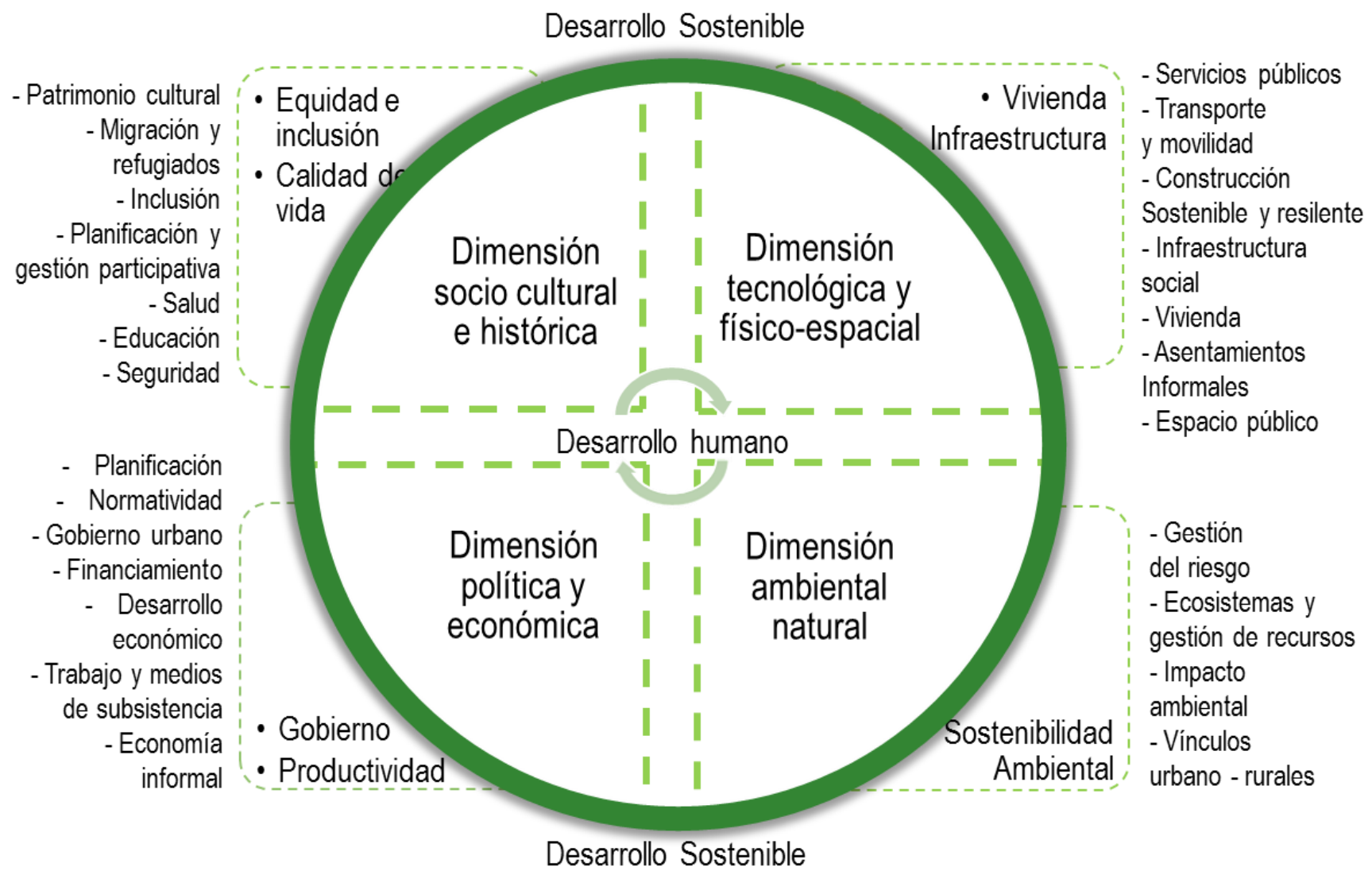

Dimensiones, temas y subtemas para la observación

Elaboración propia

En busca de la mayor articulación posible con las políticas públicas, el ámbito de observación que se propone inicialmente para el OHB concuerda con la región definida por la Misión del Sistema de ciudades como la "Aglomeración de la Sabana de Bogotá", la cual comprende 23 municipios (DNP, 2012): Bogotá D.C., Bojacá, Cajicá, Chía, Cogua, Cota, Facatativá, Funza, Gachancipá, Guatavita, La Calera, Madrid, Mosquera, Nemocón, Sesquilé, Sibaté, Soacha, Sopó, Sutatausa, Tabio, Tausa, Tocancipá, Zipaquirá. 
El Observatorio busca articularse a la Red de OULs de ONU-Habitat, para lo cual, su estructuración se plantea con base en la metodología general propuesta para este programa. De igual manera, el OHB busca establecerse como el nodo Bogotá del OUC, asumiendo para ello la utilización del Índice de Prosperidad Urbana de Naciones Unidas. En cuanto al sistema de información, se prevé la utilización del software diseñado por ONU-Hábitat para los Observatorios que conforman la red de OULs y, de ser necesario, se planea la implementación de un sistema de información propio que se corresponda con las especificidades del OHB.

Para el proceso de georeferenciación, se plantea el aprovechamiento de las capacidades ya instaladas en el laboratorio de Georeferencia de la Facultad de Arquitectura de la UGC.

Para la articulación del OHB con las funciones sustantivas que desarrolla la Facultad de Arquitectura se prevé, entre otras, las siguientes estrategias: vinculación de los estudiantes de los cursos del área de investigación al desarrollo de las actividades propias del Observatorio y uso de la información por este producida para el apoyo de los cursos de las demás áreas; vinculación de los grupos de investigación de la facultad de Arquitectura y de otras facultades de la UGC, con sus respectivos semilleros de investigación, como parte del área investigativa del OHB; organización de eventos académicos de discusión de las temáticas observadas como escenarios complementarios al plan de estudios; desarrollo de procesos de consultoría, vinculando de esta manera la investigación y la extensión.
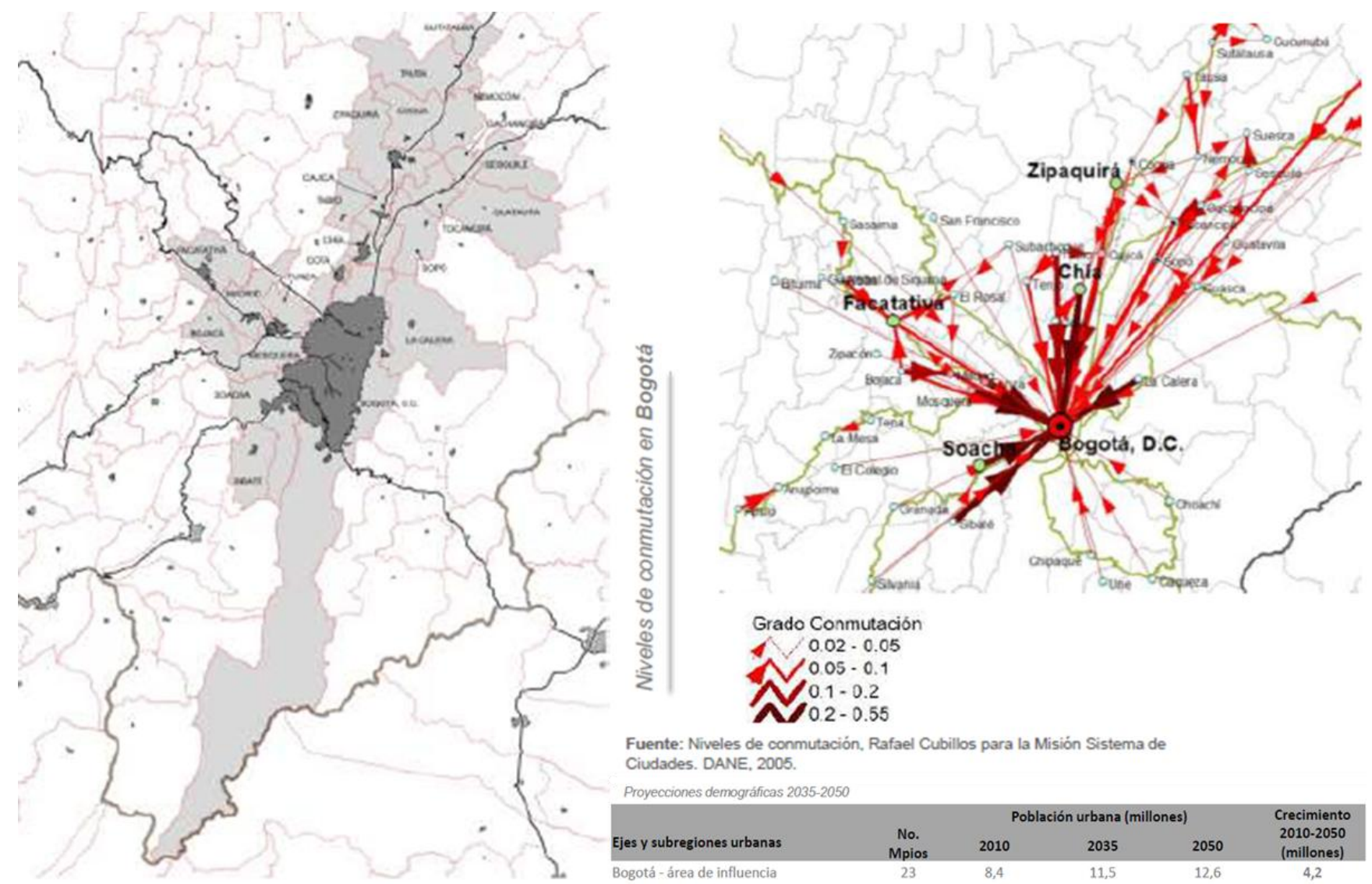

Aglomeración de la Sabana de Bogotá (DNP, 2012) 


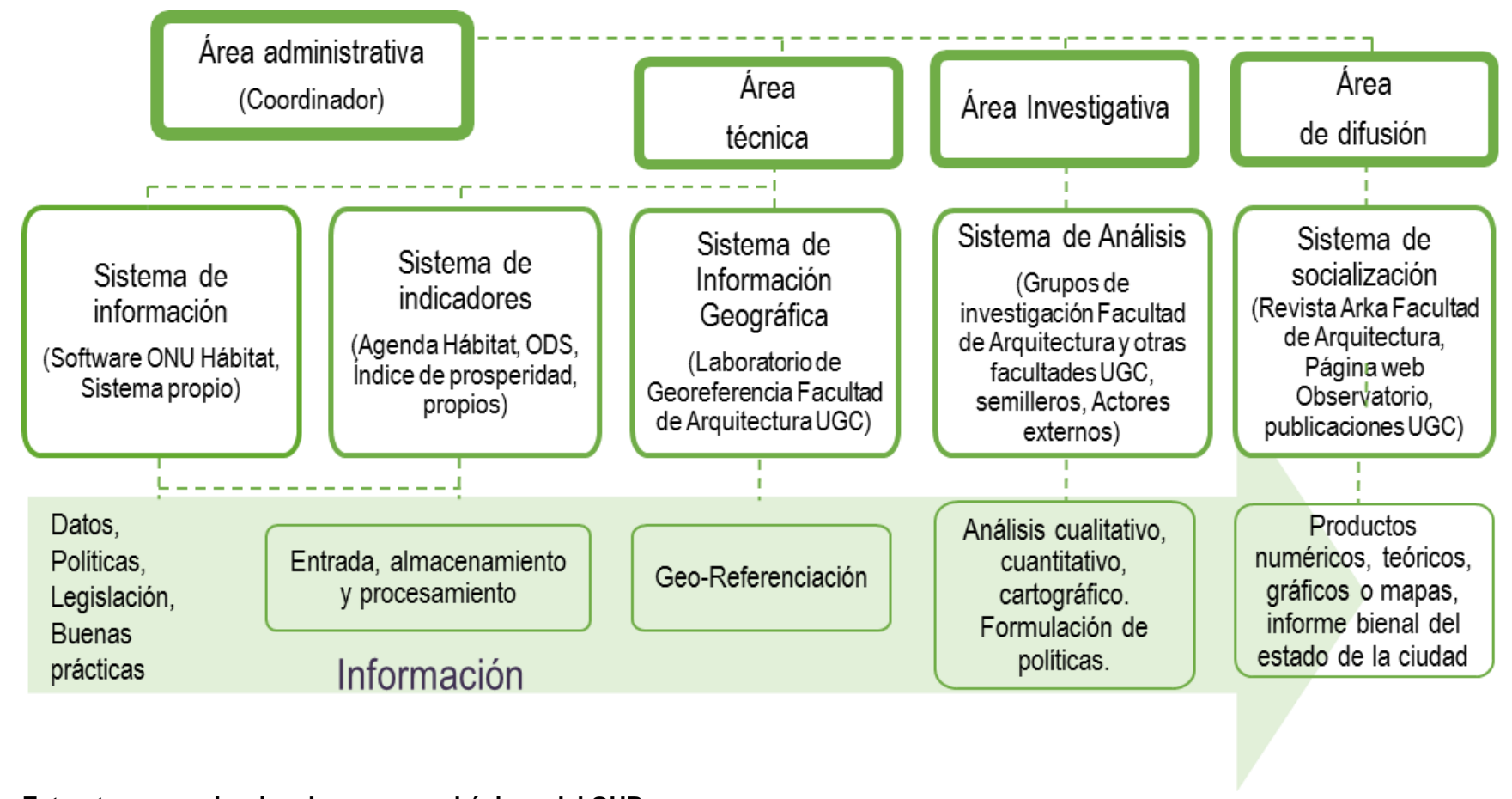

Estructura organizacional y procesos básicos del OHB Elaboración propia

\section{CONCLUSIONES}

Además de adolecer de un observatorio cuyo trabajo permita comparar a la ciudad región en un contexto internacional y evaluar su situación y avances respecto a lo planteado en agendas multilaterales, en Bogotá los observatorios existentes, al funcionar enfocados en diferentes temas y en cabeza de distintas entidades del gobierno de la ciudad y por su naturaleza en la mayoría de los casos netamente pública, no logran una gestión suficiente, confiable, imparcial e integrada de la información acerca de las distintas dimensiones y escalas que en conjunto conforman el hábitat.

Es claro que la virtud de la metodología propuesta por ONU-Hábitat para la creación de Observatorios vinculados a la red de OULs, es poder comparar a las diversas ciudades, monitorear los esfuerzos para el desarrollo de los consensos y los compromisos establecidos, desde las diferentes dimensiones del hábitat. Sin embargo, al también ser evidente que el conjunto de indicadores y las metodologías de estimación elaborados por esta organización, así como aquellos propuestos para el seguimiento de los ODS, son un planteamiento genérico, resulta necesario que éstos sean acercados a las especificidades de la ciudad-región y que a través de foros de discusión se establezcan indicadores adicionales que atiendan a las prioridades del ámbito de observación y a los intereses propios de la UGC y su Facultad de Arquitectura. En este sentido, el problema de la informalidad podría constituirse en uno de los temas en los que se haga mayor énfasis, teniendo en cuenta la inexistencia de un observatorio sobre el tema, la importancia de la cuestión en la ciudad, el interés de la UGC y su Facultad de Arquitectura en las poblaciones vulnerables y el antecedente de Observatorio en la misma Facultad; por las mismas razones, en temas como el Patrimonio, la renovación urbana y la vivienda social, el OHB podría fijar también su atención.

Las experiencias de Metrosum y el OUC muestran que, además, resulta fundamental que en las primeras etapas de implementación del Observatorio, se cree una red de distintos actores interesados en el tema como plataforma de apoyo social para el incremento del efecto y la utilidad real de la organización. Así mismo, que en la fase inicial se establezca un protocolo para el monitoreo, se definan escalas geográficas de análisis con sus respectivas coberturas para el SIG y se firmen convenios con distintas entidades para el acceso a la información. 


\section{BIBLIOGRAFÍA}

D.N.P., Departamento Nacional de Planeación. (2012). Misión para el fortalecimiento del sistema de ciudades. Bogotá: Autor.

FACULTAD DE ARQUITECTURA. (2010). Subsistema de investigación y fundamentación de la línea central y las líneas primarias. Bogotá: Universidad La Gran Colombia.

- (2011): Subsistema de Proyección Social. Bogotá: Universidad La Gran Colombia.

- (2016): Proyecto Educativo del Programa de Arquitectura. Bogotá: Universidad La Gran Colombia.

FRAUSTO, O., SOIJET, M., \& CHACÓN, L. (2013). La observación urbana: experiencias de México, Argentina y Costa Rica. En O. FRAUSTO y J. ROJAS LÓPEZ (comps.), La observación urbana en ciudades latinoamericanas (13-31). Chetumal: Universidad de Quintana Roo.

GALINDO VERGARA, L. M. (2012). El paradigma del Desarrollo Urbano Sostenible -DUS- como referente del proceso de toma de decisiones legislativas en Colombia. (Tesis de Maestría). Bogotá: Universidad del Rosario.

HABITAT, I. I. (1996). Declaración de Estambul sobre Asentamientos Humanos-.Agenda Hábitat-, UNCHS.

MARÍN VILLEGAS, J. C. (2014). Análisis de la habitabilidad de un proyecto de vivienda social multifamiliar en el marco de procesos de reasentamiento poblacional. (Tesis de Maestría). Manizales: Universidad Nacional de Colombia.

ONU-HABITAT COLOMBIA. (2015). 1er Reporte sobre el estado de las ciudades de Colombia: camino hacia la prosperidad urbana. Bogotá: ONU-Habitat Colombia.

ONU-HABITAT COSTA RICA. (2010). Guía para la multiplicación de observatorios locales. San José: ONUHabitat Costa Rica.

ONU-HABITAT y SEDESOL, Secretaría de Desarrollo Social. (2012). Guía metodológica Constitución y operación de las Agencias de Desarrollo Urbano y los Observatorios Urbanos Locales. México D.F.: Autor.

SÁNCHEZ, C. E. (2003). En búsqueda de una episteme. Bogotá: Universidad Nacional de Colombia.

STRONG, M. (1998). Cumbre para la Tierra, Programa 21. Nueva York: Naciones Unidas.

UNIVERSIDAD LA GRAN COLOMBIA. (2016a). Proyecto Educativo Institucional "Forjadores de la nueva civilización". Bogotá: Autor.

- (2016b): Plan Estratégico institucional de Desarrollo (PEID) 2016 - 2020. Bogotá: Autor.

VICEPRESIDENCIA DE LA REPÚBLICA DE COLOMBIA. (2006). Guía para conformación de observatorios de $D H$ y DIH. Bogotá: autor.

WORLD COMMISSION ON ENVIRONMENT AND DEVELOPMENT. (1992). Nuestro Futuro Común. Madrid: Autor.

Fuentes electrónicas:

CHÁVEZ, T., ÁLVAREZ, J. A. y GARROCHO, C. (2007). El observatorio metropolitano de Toluca: lecciones, propuestas y desafíos. Economía, Sociedad y Territorio, 7(25), 157-214. http://www.redalyc.org/articulo.oa?id=11102507 (Consulta: 12/06/2016).

COLOMBIA. MINISTERIO DEL INTERIOR. Decreto No.3680 de 2011, Octubre 04, por el cual se reglamenta la Ley 1454 de 2011. Bogotá: Autor.

http://camacol.co/sites/default/files/base_datos_juridico/DECRETO\%20MININTERIOR\%20NACIONAL\%203680 \%202011.pdf (Consulta: 23/05/2016).

CORREA ASSMUS, G. y CASTELLANOS LONDOÑO, I. (2014). Observatorios académicos: hacia una cultura en el uso de la información. Revista Universidad de La Salle, 0(64), 131-140. http://revistas.lasalle.edu.co/index.php/ls/article/view/3230/2574 (Consulta: 23/04/2016).

INDICATORS, S. D. S. N. (2015). A monitoring framework for sustainable development goals: launching a data revolution for the SDGs. Sustainable Development Solutions Network.

http://unsdsn.org/wp-content/uploads/2015/05/FINAL-SDSN-Indicator-Report-WEB.pdf (Consulta 16/06/2016).

MARCIAL, N. A. (2009). ¿Qué son los observatorios y cuáles son sus funciones?. Revista Innovación Educativa, 9(47), 5-17.

http://www.redalyc.org/articulo.oa?id=179414895002 (Consulta: 15/03/2016).

METROSUM, Observatorio Metropolitano de Toluca. (s.f.). Misión del Observatorio. Toluca. http://www.metrosum.org.mx/index.php/mision (Consulta 05/02/2016)

ONU-HABITAT. $\quad$ (s.f.). Instalación un de Observatorio urbano. http://www.onuhabitat.org/index.php?option=com_docman\&task=doc_details\&gid=83\&ltemid=71. (Consulta: 18/08/2016). 
ONU-HABITAT. (2016). Borrador Cero de la Nueva Agenda Urbana. http://www.habitat3.org/zerodraft (Consulta: 10/09/16).

ONU-HABITAT COLOMBIA. (2016). Hacia un observatorio urbano nacional en Colombia. Bogotá: autor. http://www.onuhabitat.org/ (Consulta: 08/09/16).

ONU-HABITAT / ROLAC. (2004). Guía de aplicación: sistema integrado de indicadores urbanos observatorio de medio ambiente urbano. Río de Janeiro: ONU-hábitat / Rolac. http://www.onuhabitat.org/index.php?option=com_docman\&task=doc_download\&gid=85\&ltemid=71 (Consulta: 18/05/16).

O.U.C., Observatorio Urbano de Córdoba. (s.f.). Ficha técnica. Córdoba.

http://ouc.unc.edu.ar/ficha-tecnica/ (Consulta 05/02/2016)

PARDO, T. S. y MÉNDEZ, L. (2014). Causas comunes. Observatorio urbano: modelo de responsabilidad social universitaria. Revista Universidad de La Salle, $0(64), \quad 53-80$. http://revistas.lasalle.edu.co/index.php/ls/article/view/3227/2571 (Consulta: 23/04/2016). 